\title{
Emploi des radiations ionisantes en technologie laitière
}

\author{
par \\ J. MORRE*, L. SERRES** et F. JANIN***
}

L'emploi des rayonnements est depuis la découverte de F. Appert, il y a 160 ans environ, la seule technique vraiment nouvelle pour stériliser ou pasteuriser les aliments, produits laitiers compris.

Leur intérêt est de traiter l'aliment dans son emballage définitif, sans élever la température du produit.

Mais leur emploi est freiné, car souvent aux doses utiles il se développe dans l'aliment des odeurs et des goûts anormaux.

La suspicion qui s'attache au domaine nucléaire, a ralenti sinon empêché la délivrance d'autorisations d'emploi. En France seul est autorisé l'emploi des radiations comme antigerminatif pour les pommes de terre, les oignons et les aulx et pour stériliser les provendes pour animaux axéniques, c'est-à-dire élevés sans germes. Même la stérilisation des instruments médicaux en plastique à usage unique a dû faire l'objet d'une autorisation.

\section{Rayonnements utilisés et unités}

Les rayonnements utilisés sont les rayons $\beta, \gamma$ et $X$ et rarement le rayonnement protonique, c'est-à-dire composé de noyaux d'hydrogène.

Les unités sont: le rad (multiples Krad et Mrad soit $10^{3}$ et $10^{6}$ rads) pour la dose absorbée quel que soit le rayonnement, la Curie, qui représente une vitesse de désintégration, c'est-à-dire l'activité d'une source. Enfin le $\mathrm{MeV}$ ou million d'électron-volt qui mesure l'énergie, donc le pouvoir de pénétration.

* Docteur vétérinaire et Docteur ès sciences.

** Directeur adjoint, Ingénieur agronome.

*** Attachée de Recherches.

Laboratoire Central d'Hygiène Alimentaire (Dr Pantaléon, Directeur), 43, rue de Dantzig, 75015 Paris. 


\section{Irradiateurs}

Le traitement a lieu soit dans des accélérateurs d'électrons, soit dans des irradiateurs à radioéléments. Les premiers délivrent des doses importantes en peu de temps, mais la pénétration est faible, les deuxièmes ont un débit de dose limité, mais une excellente pénétration. Ces derniers utilisent les radioéléments produits en abondance dans les piles atomiques, le colbalt 60 en particulier.

Il apparaît en U.R.S.S. et aux U.S.A. des irradiateurs spécialisés pour le lait liquide et les emballages en carton. Le lait par un mouvement de rotation rapide, prend la forme d'un film cylindrique. La source de rayonnement est au centre et la dose délivrée est très homogène.

\section{Emploi en industrie laitière}

Si la stérilisation du lait et sa pasteurisation par les ondes hertziennes ou les infra-rouges offrent une grande sécurité et si les ultraviolets sont prometteurs, les rayonnements ionisants stérilisent bien le lait, mais font apparaître dès la dose de $50 \mathrm{Krad}$ selon certains auteurs, des modifications de couleur, d'odeur, de goût, qui rendent la méthode inutilisable, il apparaît des odeurs et des saveurs de corne brûlée.

Chimiquement il y a production d'allyle-mercaptants et de sulfite d'allyle. Des radicaux - $\mathrm{SH}$ apparaissent et croissent avec la dose.

Des améliorations technologiques sont envisageables pour diminuer ces accidents : irradier à basse température, sous vide, en atmosphère inerte : $\mathrm{CO}^{2}$ ou $\mathrm{N}$, à l'état de lait sec ou par emploi de protons, qui semblent donner de bons résultats.

Pien propose l'irradiation du lait pour le pasteuriser. Aux doses de 0,1 à 0,5 Mrad, il n'y a pas d'altérations. L'intérêt est évident : le traitement a lieu à froid dans les récipients définitifs de vente. Pour la stérilisation, il propose une technique de tyndallisation: une thermopasteurisation à $75^{\circ} \mathrm{C}$, le temps d'homogénéiser et de désaérer le lait, suivie d'une incubation de 2 heures à $35^{\circ} \mathrm{C}$ et d'une radiopasteurisation à une dose de $0,4 \mathrm{Mrad}$ à fixer exactement. La première pasteurisation tue les formes végétatives des germes. Lors de l'incubation, grâce à l'absence d'oxygène, les germes ne donnent pas de forme sporulée, mais uniquement des corps végétatifs, qui sont sensibles aux rayonnements. Le lait serait ainsi stérilisé sans dépasser la température de $75^{\circ} \mathrm{C}$. Pour le lactosérum, dont l'homogénéisation est inutile, deux radiopasteurisations à deux heures d'intervalle, pourraient être utilisées, la stérilisation serait obtenue sans élévation de température.

L'innocuité du lait irradié sec en poudre a été étudiée par l'équipe du Pr Diehl à Karlsruhe dans le cadre du "Projet International ». 
Des populations de rats soumis à des régimes riches en lait irradié, n'ont présenté aucun accident: même croissance, même prolificité, cancers et malformations congénitales équivalents entre les diverses populations: d'expérience ou témoins. Il semble donc que toute réticence de ce point de vue puisse être écartée. Mais cette méthode se heurte aux difficultés d'agrément des autorités sanitaires et pour un facteur moindre à l'inertie des industriels. Nous retrouverons ce problème pour la stérilisation des emballages.

Sous réserve que le goût, la saveur et l'odeur ne soient pas modifiés, la technique proposée par Pien mérite une étude et une expérimentation.

Le seul emploi actuel est médical: stérilisation du lait humain réservé aux prématurés utilisé en Grande-Bretagne aux lieu et place de la chaleur : $105^{\circ} \mathrm{C}, 60 \mathrm{mn}$; il y aurait une altération moindre des facteurs essentiels du lait: lactoferrine et protéines en particulier.

\section{Beurre et crème}

L'irradiation aux doses faibles: $10 \mathrm{Krad}$ ne produit que peu d'hydroxyperoxydes, de carbonylés et l'oxydation est ménagée. Mais aux doses de 1 à 10 Mrad, il y a production de telles modifications que le produit ne peut plus être consommé.

Ce sont les caroténoïdes et le tocophérol qui sont les plus altérés, alors que les stérols et l'acide linoléique souffrent moins. La vitamine A est détruite.

\section{Fromages}

Une dose comprise entre 0,1 et 1 Mrad pasteurise le fromage, la maturation continue. A 10 Mrad la stérilisation est totale. Mais dès $70 \mathrm{Krad}$, il apparaît une odeur désagréable et à $200 \mathrm{Krad}$ on note un goût de suif avec saveur aigre et des défauts dans la pâte.

Par contre l'irradiation de framboise à 0,36 Mrad avant leur inclusion dans le yaourt améliore beaucoup la conservation du produit. Il apparaît certes une odeur anormale, mais qui disparaît très vite.

\section{Caséine et protéine}

La stérilisation de ces produits souvent très contaminés sans élever leur température, est d'un grand intérêt. S'il apparaît un goût ou une odeur anormale, l'importance est moindre que pour le lait ou le fromage, compte tenu du mode d'utilisation de ces produits.

Du petit-lait séché de vache ou de brebis ou du caséinate de sodium irradiés à 2 Mrad sont stérilisés. A 1,5 Mrad, il y a pasteurisation, les qualités organoleptiques sont conservées, le $\mathrm{pH}$, la solu- 
bilité, le point isoélectrique, le temps de coagulation sont peu modifiés.

Si à la caséine on ajoute du glucose ou de l'amidon, il y a un effet protecteur et le résultat est amélioré. La chaleur à $100^{\circ} \mathrm{C}, 60 \mathrm{mn}$, altère plus les protéines: digestibilité, utilisation protéique, valeur biologique, que les rayonnements.

\section{Action sur les bactéries}

Dans le lait, des doses de $0,5,1$ et $1,5 \mathrm{Mrad}$ diminuent la contamination d'un facteur $10^{4}, 10^{5}$ et $10^{6}$, ce qui est considérable.

Des cultures de streptocoques lactiques lyophylisés sont plus résistantes que la solution aqueuse. Les germes sont plus sensibles en présence d'air que dans le vide et plus à une température de $30^{\circ} \mathrm{C}$ qu'à $5^{\circ} \mathrm{C}$.

L'âge de la culture joue un rôle: en période de développement 50 p. 100 des germes sont détruits à $10 \mathrm{Krad}$. La fin de la croissance logarithmique et la phase stationnaire sont beaucoup plus résistantes. Les germes en état de mitose active sont plus sensibles: fait bien connu.

Les coliformes sont détruits dans le lait cru avec une dose de 100 Krad, le Clostridium tyrobutyricum nécessite une dose de $360 \mathrm{Krad}$, de même les germes banaux. Les brucella disparaissent à $200 \mathrm{Krad}$, c'est $\mathrm{Br}$. melitensis qui est la plus résistante.

\section{Effet mutagène}

En irradiant des cultures de ferments lactiques d'une façon ménagée, on crée parmi les survivants des races possédant des qualités très supérieures à celles des parents.

Les bactéries productrices d'acide lactique acquièrent une grande activité protéolytique et peuvent être utilisées comme levures en fromagerie, la maturation s'en trouve améliorée. Au point de vue médical ces germes très résistants peuvent être employés pour lutter contre les bactéries intestinales pathogènes.

Après avoir reçu une dose de $100 \mathrm{Krad}$, d'autres germes voient leur production de diacétyl atteindre $39 \mathrm{mg}$ par litre de culture contre 3 initialement. Ils sont utilisés pour ensemencer le lait utilisé en margarinerie.

Pour certains germes la production de nisine, polypeptide à action antibiotique sur les Clostridium tyrobutyricum, qui causent des pertes sévères en fromagerie, passe de 470 UI à 1700 et même 2000 UI.

Les caractères acquis par irradiation sont héréditaires et se conservent au cours des cultures. 


\section{Enzymes}

Les enzymes sont moins sensibles que les bactéries aux radiations : aussi il est possible de les pasteuriser alors que le traitement par la chaleur est difficile.

Certaines enzymes du lait sont détruites partiellement : amylase, phosphatase acide, peroxydase, d'autres voient leur action exacerbée: catalase, lipase, phosphatase alcaline, ceci est dû au fractionnement des protéines sous l'action des rayonnements.

\section{La présure}

De la présure préparée à partir de Mucor pusillus est irradiée aux rayons $\mathrm{X}$ dans un tampon de phosphate $0,1 \mathrm{M}$ à $20^{\circ} \mathrm{C}$. L'enzyme est peu à peu inactivée, mais celle qui reste garde toutes ses propriétés.

Un dialysat sur cellophane irradié à 3 Mrad ne perd aucune activité, la perte est de 56 p. 100 pour $10 \mathrm{Mrad}$, dose très supérieure à celle nécessaire pour stériliser.

\section{La lipase}

Une dose de 0,7 Mrad réduit l'activité de 63 p. 100, mais l'addition de lait écrémé lyophylisé ou de lait homogénéisé protège l'enzyme, une dose de 3,2 Mrad est alors nécessaire.

\section{Enzymes fixées}

Une technique nouvelle consiste à fixer les enzymes pour qu'elles ne soient pas entraînées par le lait: ceci est intéressant quand la diastase coûte cher ou est rare.

On propose de déposer les enzymes sur un gel plastique puis de polymériser le tout par action d'électrons à la dose de $5 \mathrm{Mrad}$, à la manière des vernis irradiés. Le gel de glucoamylase ainsi préparé a une activité semblable à celle de l'enzyme, la fixation est parfaite et l'enzyme n'est pas altérée par aucun produit chimique : colle ou activateur de polymérisateur.

\section{Jauge d'épaisseur}

L'absorption des rayons gamma permet de mesurer la densité à épaisseur constante d'un produit liquide. Ces jauges sont utilisées en industrie laitière pour connaître l'état de concentration d'un lait. C'est le seul emploi actuel des rayonnements dans cette industrie.

\section{Stérilisation des emballages}

Les rayonnements permettent de réaliser une stérilisation parfaite des emballages et des films plastiques juste avant leur emploi. Cette 
technique serait très utile pour le conditionnement du lait UHT, les industriels français ont proposé cette méthode, mais il ne semble pas y avoir eu d'écho en laiterie.

\section{CONCLUSION}

Les industries agro-alimentaires doivent être à la pointe du progrès et connaître les nouvelles techniques, même si elles ne sont actuellement qu'au stade des essais, l'irradiation est une de ces méthodes. Les pays de l'Est s'y intéressent beaucoup.

La stérilisation du lait, du beurre, de la crème, du fromage est à rejeter. La tyndallisation proposée par Pien est à expérimenter. Le traitement des caséines et des protéines et la «création » de germes doués de propriétés nouvelles sont des méthodes d'avenir. La stérilisation des enzymes ou leur fixation doivent apporter une aide à l'industrie laitière.

L'emploi des rayonnements ne présente aucun danger pour la santé publique.

Actuellement c'est la stérilisation des emballages, qui devrait à côté des jauges gamma, être la plus rapidement réalisée.

\section{R és u m é}

L'emploi des radiations ionisantes en industrie laitière est freiné par le développement de goûts et d'odeurs indésirables dès les doses utiles de pasteurisation. Une technique de radio-tyndalisation est proposée.

Par contre c'est une technique d'avenir pour la pasteurisation des caséines et du petit lait. Leur grand intérêt est de donner naissance à des ferments lactiques à grande activité protéolytique, à production intense d'acide lactique, de nisine et de diacétyle, intéressants en fromagerie et en margarinerie.

Les enzymes : lipase et présure peuvent être débarrassées des germes qui les contaminent. Elles peuvent être fixées sur des supports plastiques polymérisés par les radiations.

Enfin une utilisation existe déjà : l'emploi de jauges d'épaisseur pour concentrer le lait et une verra le jour prochainement: la stérilisation des emballages du lait UHT, juste avant le remplissage.

50 références bibliographiques. 


\section{S u m m a ry}

The use of ionizing radiations in milk technology is restricted by the development of undesirable flavours when the level required for pasteurisation is reached. A radiotyndalisation technique is proposed.

This is nevertheless a technique of the future as far as the pasteurisation of caseins and whey is concerned. The importance of ionizing radiations lies in the formation of lactic mutants with high lactic acid, nisin and diacetyl production, which are of interest in cheese and margarine manufacture.

The enzymes: lipase and rennet can be freed from germs contaminating them. They can be fixed on plastic frames, polymerized by radiations.

Use of ionizing radiations already exists as thickness gauges for milk concentration and will exist for sterilisation of UHT milk packaging material prior to filling.

50 bibliographic references.

\section{Bibliographie}

Les références sont groupées par chapitre.

Dairy Science Abstracts $=$ D.S.A.

Industrie laitière

Costa Silva (J.). - Effects of heat in milk (Esp.). D.S.A., 1977, $\mathrm{n}^{\circ} 1006$.

ToRre (G.). - Evaluation of some radiations used for milk sterilisation (It.). D.S.A., $1973, \mathrm{n}^{\circ} 561$.

Hsu (H.) \& Coll. - Gamma irradiation effect on the sulfhydryls content of skimmilk powder (Ang.). D.S.A., 1973, $\mathrm{n}^{\circ}$ 2330-31.

Ahmed (N.U.) \& Coll. - The extreme and variable susceptibility of crystalline lactose to ionizing radiations (Ang.). D.S.A., 1973, $\mathrm{n}^{\circ} 986$.

Bүснкоv (V. P.) \& Coll. - Experimental findings on effects on foods of irradiation with protons and gamma-rays (Rus.). D.S.A., 1975, $\mathrm{n}^{\circ} 2145$.

KHoRshiD (M. A.) \& Coll. - Effect of gamma-ray irradiation on some chemical and physical properties of buffalo milk (Ang.). D.S.A., 1977, $\mathrm{n}^{\circ} 545$.

Odegov (N. I.) \& Coll. - Effects of ionizing radiation on milk (Rus.). D.S.A., 1976, n 6945.

PIEN (J.). - Les stérilisations de l'avenir. La technique laitière. 1977, 32, $\mathrm{n}^{\circ}$ 909, 15-17 et $\mathrm{n}^{\circ}$ 904-905, 7 .

Raptopoulou-Gigi (M.) \& Coll. - Antimicrobial proteins in sterilised human milk (Ang.). D.S.A., 1977, $\mathrm{n}^{\circ} 3201$.

\section{Comestibilité}

FAO/IAEA. - Information relating of the wholesomeness of irradiated food (Ang.). D.S.A., 1976, $\mathrm{n}^{\circ} 2460$,

FAO/IAEA. - The presence of malonaldehyde irradiated foodstuffs (Ang.). D.S.A., 1976, n 2461. 
RENNER (H.W.) \& Coll. - On the inocuity of hight concentrations of free radicals in irradiated foods (All.). D.S.A., 1974, $\mathrm{n}^{\circ} 2014$.

SCHERZ (H.). - Formation of malondialdehyde by irradiation of food (All.). D.S.A., 1973, n ${ }^{\circ} 1976$.

Reichelt (D. V.), Renner (H. W.) \& Diehl (J. F.). - Long-term animal feeding study for testing the wholesomeness of an irradiated died with a hight content of free radicals (All,). D.S.A., 1975, $\mathrm{n}^{\circ}$ 7161-62.

\section{Beurre et Crème}

KHorShid (M. A.). - The effect of gamma-ray irradiation on buffalo milk fat (Ang.). D.S.A., 1973, n 1952.

Stamatov (S. D.) \& Coll - Changes in IR and UV spectra and formation of geometric and positional isomers after gamma irradiation of sunflower oil, lard and butter (All.). D.S.A., 1977, $\mathrm{n}^{\circ} 559$.

Ivanov (S. A.) \& Coll. - The gamma irradiation-induced degree of oxidation, hydrolysis and polymerisation of sunflower oil, lard and butter (All.). D.S.A., $1976, \mathrm{n}^{\circ} 5897$ et 99.

WAGNER (K. H.). - Changes of foods preserved by irradiation (All.). D.S.A., $1973, \mathrm{n}^{\circ} 2322$.

\section{Fromages}

OdEGov (N. I.) \& Coll. - Effect of ionizing radiation on microbiological processes in Rossiiskii cheese (Rus.). D.S.A., 1976, $\mathrm{n}^{\circ} 7741$.

ScotT (R.). - Cheesemaking-enzymology or bacteriology ? (Ang.). D.S.A., 1973, $\mathrm{n}^{\circ} 1968$.

Kiss (I.). - Attemps to increase storage stability of strawberry yoghurt by combination treatments (Ang.). D.S.A., 1976, $\mathrm{n}^{\circ} 429$.

\section{Caséine et protéine}

Imbert-Pondaven (A.). - Etude de l'évolution des lacto-sérums au cours de leur conservation. Le Lait, 1977, $\mathrm{n}^{\circ}$ 568, 521-546.

Dimitrova (N.) \& Coll. - Reduction of bacterial contamination of protein preparations by ionizing radiation : physio-chemical and organoleptic changes (Bul.). D.S.A., 1976, n 1456.

Uмmmoto (Y.) \& Coll. - III. Digestion of gamma-irradiated casein by some proteolytic enzymes (Jap.). D.S.A., 1973, $\mathrm{n}^{\circ} 2298$.

Bychкоv (V. P.) \& Coll. - Effect of ionizing radiation on food products (Rus.). D.S.A., $1974, \mathrm{n}^{\circ} 691$.

PATEL (P. C.) \& Coll, - Effect of gamma irradiation on gel chromatographic pattern of Cottage cheese whey (Ang.). D.S.A., 1973, $\mathrm{n}^{\circ} 3177$.

SAITo (Z.). - Effects of gamma irradiation on some properties of bovine casein micelles (Ang.). D.S.A., 1976, $\mathrm{n}^{\circ} 1800$.

BRANKova (R.) \& Coll. - Reduction of bacterial contamination of protein preparation by ionizing radiation (Bul.). D.S.A., 1976, $\mathrm{n}^{\circ} 3007$.

HaRMUth-HoEne (A. E.). - The effect of non-protein food constituents on the nutritive value of radiation-sterilised casein (Ang.). D.S.A., 1977, $\mathrm{n}^{\circ} 1535$.

\section{Bactériologie}

UMANSKII (M. S.) \& Coll. - Effects of ionizing radiation on bacterial contamination of milk (Russ.). D.S.A., 1976, $\mathrm{n}^{\circ} 7361$.

NaguiB (M. M.) \& Coll. - Effect of gamma irradiation on some bacteriological and chemical properties of buffalo milk (Ang.). D.S.A., 1975, $\mathrm{n}^{\circ} 2779$.

NIEWOLAK (S.) \& Coll. - Effect of irradiation conditions: 1) on survival of Streptococcus lactis; 2) at different stages of growth (Pol.). D.S.A., 1974, $\mathrm{n}^{\circ}$ 2054-55. 
Naguib (M. M.). - Effect of gamma irradiation on the survival of Brucella species in milk (Ang.). D.S.A., 1975, $\mathrm{n}^{\circ} 336$.

Effet mutagène

Dilanian (Z.) \& Coll. - Use of X-ray and gamma induced mutants of lactic acid bacteria in the manufacture of dairy products (Ang.). D.S.A., 1976, $\mathrm{n}^{\circ} 8119$.

Shmeleva (L. I.) \& Coll. - Improvement of starter cultures by 60 Co gammairradiation (Rus.). D.S.A., 1976, $\mathrm{n}^{\circ}$ 3008, in starter quality (Rus.). D.S.A., 1977, $\mathrm{n}^{\circ} 443$.

HylmaR (B.) \& Coll. - Study on obtaining streptococcus lactis mutants with increased nisin production by gamma irradiation (Tch.). D.S.A., 1975, $\mathrm{n}^{\circ} 7187$ et 2750 .

\section{Enzymes}

SASAKI (Y.) \& Coll. - Effects of gamma irradiation on metabolic activities of food microorganismes: I. Yeasts and lactic acid bacteria (Jap.). D.S.A., $1974, \mathrm{n}^{\circ} 1126$.

IsmaIL (A. A.). - Effects of gammaray irradiation on some enzymes activities of buffalo milk (Ang.). D.S.A., 1975, $\mathrm{n}^{\circ} 8082$.

Kovacs-Proszt (G.) \& Coll. - X-ray inactivation in solution of rennin from Mucor pusillus (Ang.). D.S.A., 1975, $\mathrm{n}^{\circ} 1359$.

Bachman (S.) \& Coll. - Effects of 60 Co irradiation on the enzyme activity of rennet preparation of different purities (Pol.). D.S.A., 1973, $\mathrm{n}^{\circ} 1435$.

SAIto (Z.). - Milk lipase. - IX. Effects of gamma irradiation on lipase activities (Jap.). D.S.A., 1975, $\mathrm{n}^{\circ} 7319$.

Suzuki (H.) \& Coll. - Immobilization of enzymes by radiation polymerisation (Ang.). D.S.A., 1977, $\mathrm{n}^{\circ} 5917$.

Technologie, irradiateurs et empaquetage

Tsor (Y.) \& Coll. - Device for treating liquids by irradiation (Russ.). D.S.A., 1976, n' 4270.

Hoffman (C. R.). - New radiation processing system (Ang.). D.S.A., 1973, $\mathrm{n}^{\circ} 793$. 